\title{
Incoherent dynamics in neutron-matter interaction
}

\author{
Ludovico Lanz* and Bassano Vacchini ${ }^{\dagger}$ \\ Dipartimento di Fisica dell'Università di Milano and Istituto Nazionale di Fisica Nucleare, Sezione di Milano, \\ Via Celoria 16, I-20133, Milan, Italy
}

(Received 28 March 1997; revised manuscript received 23 July 1997)

\begin{abstract}
Coherent and incoherent neutron-matter interaction is studied inside a recently introduced approach to subdynamics of a macrosystem. The equation describing the interaction is of the Lindblad type and, using the Fermi pseudopotential, we show that the commutator term is an optical potential leading to well-known relations in neutron optics. The other terms, usually ignored in optical descriptions and linked to the dynamic structure function of the medium, give an incoherent contribution to the dynamics, which keeps diffuse scattering and attenuation of the coherent beam into account, thus warranting fulfillment of the optical theorem. The relevance of this analysis to experiments in neutron interferometry is briefly discussed.
\end{abstract} [S1050-2947(97)06111-8]

PACS number(s): 03.75.Be, 03.65.Bz, 03.65.Ca

\section{INTRODUCTION}

In recent years there has been a rapidly growing interest in the field of particle optics, especially neutron and atom optics (for a recent review see Refs. [1-5] and [6], respectively, and references quoted therein), due to a spectacular improvement of the experimental techniques, connected to the introduction of the single-crystal interferometer in the first case, and to progress in microfabrication technology and development of intense tunable lasers in the second one. Such new achievements provide very important tests verifying the validity of quantum mechanics, especially in that it predicts wavelike behaviors even for single microsystems.

At the same time a new challenge arises, linked to the accuracy required in the description of the interaction between the microsystem and the apparatus acting as optical device. The question of the description of the dynamics of a microsystem interacting with a system having many degrees of freedom (e.g., matter seen as an optical medium characterized by an index of refraction) has been extensively studied, and contains some typical quantum-mechanical features, such as quantum correlations between the two systems, by which a reduced description of the microsystem's degrees of freedom can arise only by suitable approximations. This subtle point is particularly important in the case of particle optics, where the main interest is devoted to the coherent wavelike behavior of particles, as can be justified on the basis of the similarity between a Schrödinger equation with an optical potential and the Helmholtz wave equation $[3,6]$. The very existence of such an optical description of the interaction is far from trivial, and depends strongly on the experimental conditions. Attention has been mostly devoted to exploiting the optical analogies, while little has been said about the borderline between the optical regime, in which coherent effects are predominant and a classical wavelike description plays a major role, and an incoherent regime, where incoherent effects, caused by the interaction between

\footnotetext{
*Electronic address: lanz@mi.infn.it

†Electronic address: vacchini@mi.infn.it
}

the microsystem and the apparatus and showing typical particlelike features, should not be neglected. This attitude is exemplified in neutron optics by the use of the "coherent wave" formalism, instead of a reduced density-matrix description, as usually adopted in quantum optics.

In this paper we want to address the question of how to describe both regimes consistently, applying a recently developed approach to the description of irreversible subdynamics in quantum mechanics [7-9] to the specific case of neutron-matter interaction. In this approach the use of an effective $T$ matrix describing the local interactions as practical starting point leads to the introduction of a time scale and in the particular case of particle-matter interaction to a dynamical semigroup, whose generator has the typical Lindblad form [10]. The expressions appearing in the generator are linked to particle-particle interactions, like the Fermi pseudopotential, and to properties of the macroscopic system, like the dynamic structure function, first introduced by van Hove [11]. The first part of the generator accounts for the description of the coherent interaction in terms of optical potential and index of refraction well known in neutron optics $[3,12,13]$. The remaining part is shown to be related to the dynamic structure function or, equivalently, to the density correlation function, and leads in a straightforward way to results obtained in the so-called "rigorous theory of dispersion" [3].

The paper is organized as follows: in Sec. II we give an account of the formalism; in Sec. III it is applied to neutron optics; in Sec. IV we consider diffuse scattering, the connection to the dynamic structure function, and fulfillment of the optical theorem; in Sec. V we evaluate possible experimental consequences; in Sec. VI we comment on our results indicating potential future developments.

\section{INTRODUCTION OF THE FORMALISM}

In this section we briefly introduce the formal scheme, restricted to a description of a microsystem following Ref. [7], to which we refer the reader for further details. We indicate by $\mathcal{H}^{(1)}$ the Hilbert space in which the microsystem is to be described; its energy eigenvalues are $E_{f}$, with energy 
eigenstates $u_{f}$, spanning $\mathcal{H}^{(1)}$. Both systems will be considered confined, e.g., in a box. We shall adopt the second quantization formalism, setting, for the Hamiltonian $H$ of the system,

$$
H=H_{0}+H_{m}+V, \quad H_{0}=\sum_{f} E_{f} a_{f}^{\dagger} a_{f}, \quad\left[a_{f}, a_{g}^{\dagger}\right]_{\mp}=\delta_{f g},
$$

where $a_{f}$ is the destruction operator for the microsystem, either a Fermi or a Bose particle, in the state $u_{f}$, and $H_{m}$ is the Hamilton operator for the sole macrosystem $\left(\left[H_{m}, a_{f}\right]=0\right)$. Indicating by $\mathcal{H}_{F}$ the whole Fock space, and by $\mathcal{H}_{F}^{0}$ its subspace in which $N=\Sigma_{h} a_{h}^{\dagger} a_{h}$, the number of microsystems, is equal to zero, we will denote with $|\lambda\rangle$ the basis of eigenstates of $H_{m}$ spanning $\mathcal{H}_{F}^{0}, H_{m}|\lambda\rangle=E_{\lambda}|\lambda\rangle$, and $N|\lambda\rangle=0 . V$ represents the interaction potential between the two systems. Having it in mind to describe situations in which only one particle is observed in each experimental run, or equivalently a collection of noninteracting particles in each run, we assume for the statistical operator the expression

$$
\varrho=\sum_{g f} a_{g}^{\dagger} \varrho^{m} a_{f} \varrho_{g f},
$$

where $\varrho^{m}$ is a statistical operator in the subspace $\mathcal{H}_{F}^{0}$, representing the macrosystem, and, therefore

$$
a_{f} \varrho^{m}=0 \quad \varrho^{m} a_{f}^{\dagger}=0 \quad \forall f,
$$

while $\varrho$ is a statistical operator in the subspace $\mathcal{H}_{F}^{1}$ of $\mathcal{H}_{F}$, in which $N=1$. The coefficients $\varrho_{g f}$ build a positive, trace-1 matrix, which can be considered as the representative of a statistical operator $\hat{\varrho}$ in $\mathcal{H}^{(1)}$. Being interested in the subdynamics of the microsystem we shall exploit the following reduction formula, valid for any operator of the form $A=\Sigma_{f, g} a_{f}^{\dagger} A_{f g} a_{g}=\Sigma_{f, g} a_{f}^{\dagger}\langle f|\hat{\mathrm{A}}| g\rangle a_{g}$ :

$$
\operatorname{Tr}_{\mathcal{H}_{F}}(A \varrho)=\sum_{f, g} A_{f g} \varrho_{g f}=\operatorname{Tr}_{\mathcal{H}^{(1)}}(\hat{\mathrm{A}} \hat{\varrho})
$$

We wish to determine the equation driving the time evolution of the statistical operator on a time scale $\tau$ much longer than the typical duration of microphysical interactions for the macrosystem, and therefore we shall approximate $d \varrho_{g f} / d t$ by

$$
\begin{aligned}
\frac{\Delta_{\tau} \varrho_{g f}(t)}{\tau} & =\frac{1}{\tau}\left[\varrho_{g f}(t+\tau)-\varrho_{g f}(t)\right] \\
& =\frac{1}{\tau}\left[\operatorname{Tr}_{\mathcal{H}_{F}}\left(a_{f}^{\dagger} a_{g} e^{-i / \hbar H \tau} \varrho(t) e^{i / \hbar H \tau}\right)-\varrho_{g f}(t)\right] .
\end{aligned}
$$

To proceed further, we will exploit the cyclicity of the trace operation, shifting the time evolution on the destruction and creation operators, thus working in the Heisenberg picture. In this way, no simplifying assumption is made on the structure of $\varrho^{m}$. We now introduce the following superoperators, that is to say mappings acting on the algebra generated by creation and destruction operators:

$$
\mathcal{H}^{\prime}=\frac{i}{\hbar}[H, \cdot], \quad \mathcal{H}_{0}^{\prime}=\frac{i}{\hbar}\left[H_{0}+H_{m}, \cdot\right], \quad \mathcal{V}^{\prime}=\frac{i}{\hbar}[V, \cdot] .
$$

Making use of these mappings we evaluate $e^{\mathcal{H}^{\prime} \tau}\left(a_{h}^{\dagger} a_{k}\right)$ with the aid of the following integral representation:

$$
\begin{gathered}
e^{\mathcal{H}^{\prime} \tau} a_{k}=\int_{-i \infty+\eta}^{+\infty \infty+\eta} \frac{d z}{2 \pi i} e^{z \tau}\left(z-\mathcal{H}^{\prime}\right)^{-1} a_{k}, \\
e^{\mathcal{H}^{\prime} \tau}\left(a_{h}^{\dagger} a_{k}\right)=\left(e^{\mathcal{H}^{\prime} \tau} a_{h}^{\dagger}\right)\left(e^{\mathcal{H}^{\prime} \tau} a_{k}\right) .
\end{gathered}
$$

Let us stress at this point the relevance of the formalism of second quantization. The operator quantities of interest can be expressed in terms of products of creation and destruction operators. The study of their time evolution may thus be reconducted to evaluate field operators of the form $e^{\mathcal{H}} \tau_{h}^{\dagger}$ connecting, in Fock space, subspaces with $n$ and $n+1$ particles (and similarly for $e^{\mathcal{H}^{\prime}} a_{k}$, connecting subspaces with $n$ and $n-1$ particles). Thus, even recovering at the end the usual one-particle quantum mechanics, the Fock space structure plays a central role, and accounts for the similarities between this simple case and the description of macroscopic systems $[8,9]$. For the mappings defined in Eq. (2.1), identities hold that are reminiscent of the usual ones in scattering theory:

$$
\begin{aligned}
\left(z-\mathcal{H}^{\prime}\right)^{-1} & =\left(z-\mathcal{H}_{0}^{\prime}\right)^{-1}\left[1+\mathcal{V}^{\prime}\left(z-\mathcal{H}^{\prime}\right)^{-1}\right] \\
& =\left[1+\left(z-\mathcal{H}^{\prime}\right)^{-1} \mathcal{V}^{\prime}\right]\left(z-\mathcal{H}_{0}^{\prime}\right)^{-1}
\end{aligned}
$$

In particular, we can introduce the superoperator $\mathcal{T}(z)$,

$$
\mathcal{T}(z) \equiv \mathcal{V}^{\prime}+\mathcal{V}^{\prime}\left(z-\mathcal{H}^{\prime}\right)^{-1} \mathcal{V}^{\prime}
$$

satisfying

$$
\left(z-\mathcal{H}^{\prime}\right)^{-1}=\left(z-\mathcal{H}_{0}^{\prime}\right)^{-1}+\left(z-\mathcal{H}_{0}^{\prime}\right)^{-1} \mathcal{T}(z)\left(z-\mathcal{H}_{0}^{\prime}\right)^{-1},
$$

and

$$
\mathcal{T}(z)=\mathcal{V}^{\prime}+\mathcal{V}^{\prime}\left(z-\mathcal{H}_{0}^{\prime}\right)^{-1} \mathcal{T}(z),
$$

corresponding to the Lippman-Schwinger equation for the $T$ matrix. Taking into account the fact that $[H, N]=0$ one can see that the restriction to $\mathcal{H}_{F}^{1}$ of the operator $\mathcal{T}(z) a_{k}$ has the simple general form

$$
i \hbar \mathcal{T}(z) a_{k_{\mid \mathcal{H}_{F}^{1}}}=\sum_{h} T_{h}^{k}(i \hbar z) a_{h}
$$

where $T_{h}^{k}(z)$ is an operator in the subspace $\mathcal{H}_{F}^{0}$. This restriction is the only part of interest to us, since we are considering a single microsystem. Our formalism points to this matrix, whose entries are operators on the Hilbert space of the macrosystem, as the basic mathematical tool to describe the physics of the microsystem: we will show that it yields all relevant quantities and, in our opinion, could be a sound starting point for phenomenological assumptions. $T_{h}^{k}(z)$ bears a connection to scattering theory, as it is clear from Eq. (2.4); it is also related to the thermodynamics of the macrosystem, being an operator on $\mathcal{H}_{F}^{0}$. To help clarify this con- 
nection, we consider a simple case in which $T_{h}^{k}(z)$ can be explicitly calculated. Let the macrosystem be composed of free particles

$$
H_{m}=\sum_{\eta} E_{\eta} b_{\eta}^{\dagger} b_{\eta}, \quad V=\sum_{p, \xi, q, \eta} a_{p}^{\dagger} b_{\xi}^{\dagger} a_{q} b_{\eta} V_{p \xi q \eta}
$$

where $b_{\eta}^{\dagger}$ is the creation operator of a particle in an eigenstate $v_{\eta}$ with energy $E_{\eta}$ (either a Bose or a Fermi particle). Recalling that we are describing a single particle and exploiting the superoperators introduced in Eqs. (2.1), (2.2), and (2.3), we can calculate $T_{h}^{k}(z)$ as defined by Eq. (2.5). To do this we bring to normal order the creation and destruction operators associated with the macrosystem, and restrict ourselves to a one-mode dynamics, in which, apart from statistical corrections, only one creation and one destruction operator of the type $b$ appear: that is to say, we neglect threeparticle collisions. Then one obtains

$$
\begin{aligned}
T_{f}^{k}\left(E_{k}+i \varepsilon\right)= & \sum_{\xi, \eta} b_{\xi}^{\dagger}\langle k, \xi| V^{(2)} \\
& +V^{(2)} \frac{1}{E_{\xi}+E_{k}+i \varepsilon-\left(H_{0}^{(2)}+V_{L}\right)} V_{L}|f, \eta\rangle b_{\eta},
\end{aligned}
$$

where $\varepsilon$ is a positive quantity and the following relationships hold:

$$
\begin{aligned}
\left\langle k, \xi\left|H_{0}^{(2)}\right| f, \eta\right\rangle & =\left(E_{f}+E_{\eta}\right) \delta_{k f} \delta_{\xi \eta} \\
\left\langle k, \xi\left|V_{L}\right| f, \eta\right\rangle & =\left(1 \pm b_{\xi}^{\dagger} b_{\xi}\right)\left\langle k, \xi\left|V^{(2)}\right| f, \eta\right\rangle \\
& =\left(1 \pm b_{\xi}^{\dagger} b_{\xi}\right) V_{k \xi f \eta} ;
\end{aligned}
$$

here the superscript (2) denotes operators in the two-particle Hilbert space, and statistical corrections for scattering in the medium are taken into account in the potential term $V_{L}$, implicitly defined by Eq. (2.6) and by the usual resolvent series (the + and - signs stand for Bose and Fermi statistics, respectively). The connection to the familiar $T$ matrix is evident.

We now come to the master equation describing the irreversible time evolution of the statistical operator on the chosen time scale:

$$
\begin{aligned}
\frac{d \varrho_{k h}}{d \tau}= & -\frac{i}{\hbar}\left(E_{k}-E_{h}\right) \varrho_{k h}-\frac{i}{\hbar} \sum_{f} Q_{k f} \varrho_{f h}+\frac{i}{\hbar} \sum_{g} \varrho_{k g} Q_{h g}^{*} \\
& +\frac{1}{\hbar} \sum_{\substack{f g \\
\lambda \xi}}\left(L_{\lambda \xi}\right)_{k f} \varrho_{f g}\left(L_{\lambda \xi}\right)_{h g}^{*},
\end{aligned}
$$

from which we can read off the structure of the generator of the semigroup driving the time evolution. The quantities appearing in Eq. (2.7) are defined in the following ways:

$$
\begin{gathered}
Q_{k f}=\operatorname{Tr}_{\mathcal{H}_{F}}\left[T_{f}^{k}\left(E_{k}+i \varepsilon\right) \varrho^{m}(\tau)\right], \\
Q_{h g}^{*}=\operatorname{Tr}_{\mathcal{H}_{F}}\left[T_{g}^{h \dagger}\left(E_{h}+i \varepsilon\right) \varrho^{m}(\tau)\right], \\
\left(L_{\lambda \xi}\right)_{k f}=\sqrt{2 \varepsilon \pi_{\xi}} \frac{\left\langle\lambda\left|T_{f}^{k}\left(E_{k}+i \varepsilon\right)\right| \xi(t)\right\rangle}{E_{k}+E_{\lambda}-E_{f}-E_{\xi}-i \varepsilon},
\end{gathered}
$$

with $\varepsilon$ a positive constant and $\xi(\tau)$ a complete system of eigenvectors of $\varrho^{m}(\tau)$ with eigenvalues $\pi_{\xi(\tau)}$. If we now introduce in $\mathcal{H}^{(1)}$ the operators $\hat{\mathrm{H}}_{0}, \hat{\mathrm{Q}}, \hat{\mathrm{L}}_{\lambda \xi}$, and $\hat{\varrho}$,

$$
\begin{gathered}
\left\langle g\left|\hat{\mathrm{H}}_{0}\right| f\right\rangle=E_{f} \delta_{g f}, \quad\langle g|\hat{\mathrm{Q}}| f\rangle=Q_{g f}, \\
\left\langle g\left|\hat{\mathrm{L}}_{\lambda \xi}\right| f\right\rangle=\left(L_{\lambda \xi}\right)_{g f}, \quad\langle g|\hat{\varrho}| f\rangle=\varrho_{g f},
\end{gathered}
$$

Eq. (2.7) becomes

$$
\begin{aligned}
\frac{d \hat{\varrho}(\tau)}{d \tau}= & -\frac{i}{\hbar}\left[\hat{\mathrm{H}}_{0}+\hat{\mathrm{H}}_{\mathrm{eff}}, \hat{\varrho}(\tau)\right]-\frac{1}{\hbar}\{\hat{\Gamma}, \hat{\varrho}(\tau)\} \\
& +\frac{1}{\hbar} \sum_{\xi, \lambda} \hat{\mathrm{L}}_{\lambda \xi} \hat{\varrho}(\tau) \hat{\mathrm{L}}_{\lambda \xi}^{\dagger},
\end{aligned}
$$

where

$$
\hat{\mathrm{H}}_{\mathrm{eff}}=\frac{\hat{\mathrm{Q}}+\hat{\mathrm{Q}}^{\dagger}}{2}, \quad \hat{\Gamma}=i \frac{\hat{\mathrm{Q}}-\hat{\mathrm{Q}}^{\dagger}}{2} .
$$

Verification of the conservation of the trace of the statistical operator within the adopted approximations leads to the following relationship:

$$
\hat{\Gamma} \approx \frac{1}{2} \sum_{\xi, \lambda} \hat{L}_{\lambda \xi}^{\dagger} \hat{L}_{\lambda \xi}
$$

and therefore to

$$
\begin{aligned}
\frac{d \hat{\varrho}(\tau)}{d \tau}= & -\frac{i}{\hbar}\left[\hat{\mathrm{H}}_{0}+\hat{\mathrm{H}}_{\mathrm{eff}}, \hat{\varrho}(\tau)\right]-\frac{1}{\hbar}\left\{\frac{1}{2} \sum_{\xi, \lambda} \hat{\mathrm{L}}_{\lambda \xi}^{\dagger} \hat{\mathrm{L}}_{\lambda \xi}, \hat{\varrho}(\tau)\right\} \\
& +\frac{1}{\hbar} \sum_{\xi, \lambda} \hat{\mathrm{L}}_{\lambda \xi} \hat{\varrho}(\tau) \hat{\mathrm{L}}_{\lambda \xi}^{\dagger} .
\end{aligned}
$$

This master equation is a typical result of the formalism restricted to the case of a single microsystem; for the general structure, see Refs. [8,9].

Before applying Eq. (2.10) to a concrete physical situation, it can be useful to gain some further insight into the structure of the operators appearing in it. As already stated, the quantity that the formalism suggests as a natural candidate for where to place suitable phenomenological expressions is the operator $T_{f}^{k}(z)$, an operator whose trace over the Fock space for the macrosystem calculated with $\varrho^{m}$ gives the value of the $T$ matrix for scattering from state $u_{f}$ to state $u_{k}$ averaged over the state of the macroscopic system. A quite general phenomenological expression may be obtained in the following way. Suppose that $\mathcal{T}(z)$ has the form

$\mathcal{T}(z)=\frac{i}{\hbar}[V(i \hbar z), \cdot], \quad V(z)=\sum_{k \lambda f \mu} V_{k \lambda f \mu}(z) a_{k}^{\dagger} b_{\lambda}^{\dagger} a_{f} b_{\mu}$,

with $b^{\dagger}$ and $b$ the creation and destruction operators in Fock space for the macrosystem. We thus have 


$$
i \hbar \mathcal{T}(z) a_{k}=\sum_{\lambda f \mu} V_{k \lambda f \mu}(i \hbar z) b_{\lambda}^{\dagger} a_{f} b_{\mu}=\sum_{f} T_{f}^{k}(i \hbar z) a_{f},
$$

and, supposing translation invariance in the interaction kernel,

$$
\begin{aligned}
T_{f}^{k}(z)= & \sum_{\lambda \mu} b_{\lambda}^{\dagger} V_{k \lambda f \mu}(z) b_{\mu}=\int d^{3} \boldsymbol{x} \int d^{3} \boldsymbol{y} \psi^{\dagger}(\boldsymbol{x}) u_{k}^{*}(\boldsymbol{y}) \\
& \times t(z, \boldsymbol{x}-\boldsymbol{y}) u_{f}(\boldsymbol{y}) \psi(\boldsymbol{x}) .
\end{aligned}
$$

Such an ansatz amounts to introducing an effective potential which should, in the Born approximation, give the full scattering amplitude. As a result the potential term in Eq. (2.10) is linked to the scattering amplitude, as we shall see in the next paragraph, while the incoherent contribution is generally connected to the scattering cross section. To realize this let us consider the last term of Eq. (2.7), taking the proposed ansatz into account:

$$
\begin{aligned}
& \frac{2 \varepsilon}{\hbar} \sum_{\substack{\lambda, \lambda^{\prime} \\
\lambda^{\prime \prime}}} \sum_{f, g} \int d^{3} \boldsymbol{x} \int d^{3} \boldsymbol{y} u_{k}^{*}(\boldsymbol{y}) \frac{t\left(E_{k}+i \varepsilon, \boldsymbol{x}-\boldsymbol{y}\right)}{E_{k}+E_{\lambda^{\prime \prime}}-E_{f}-E_{\lambda}-i \varepsilon} u_{f}(\boldsymbol{y})\left\langle\lambda^{\prime \prime}\left|\psi^{\dagger}(\boldsymbol{x}) \psi(\boldsymbol{x})\right| \lambda\right\rangle \\
& \quad \times\left\langle\lambda\left|\varrho^{m}(\tau)\right| \lambda^{\prime}\right\rangle \varrho_{f g}(\tau) \int d^{3} \boldsymbol{x}^{\prime} \int d^{3} \boldsymbol{y}^{\prime}\left\langle\lambda^{\prime}\left|\psi^{\dagger}\left(\boldsymbol{x}^{\prime}\right) \psi\left(\boldsymbol{x}^{\prime}\right)\right| \lambda^{\prime \prime}\right\rangle u_{g}^{*}\left(\boldsymbol{y}^{\prime}\right) \frac{t^{*}\left(E_{h}+i \varepsilon, \boldsymbol{x}^{\prime}-\boldsymbol{y}^{\prime}\right)}{E_{h}+E_{\lambda^{\prime \prime}}-E_{g}-E_{\lambda^{\prime}}+i \varepsilon} u_{h}\left(\boldsymbol{y}^{\prime}\right),
\end{aligned}
$$

and let us specialize to the case of a diagonal matrix element. Supposing the statistical operator for the microsystem is quasidiagonal and the macrosystem is at equilibrium, so that $\varrho^{m}|\lambda\rangle=\varrho_{\lambda}^{m}|\lambda\rangle$, we exploit the usual representation for the $\delta$ function, thus obtaining:

$$
\begin{aligned}
& \sum_{f} \sum_{\lambda \lambda^{\prime}} \frac{2 \pi}{\hbar} \delta\left(E_{k}+E_{\lambda}-E_{f}-E_{\lambda^{\prime}}\right) \mid \int d^{3} \boldsymbol{x} \int d^{3} \boldsymbol{y} u_{k}^{*}(\boldsymbol{y}) \\
& \times\left.\left\langle\lambda\left|\psi^{\dagger}(\boldsymbol{x}) t\left(E_{k}+i \varepsilon, \boldsymbol{x}-\boldsymbol{y}\right) \psi(\boldsymbol{x})\right| \lambda^{\prime}\right\rangle u_{f}(\boldsymbol{y})\right|^{2} \varrho_{\lambda^{\prime}}^{m} \varrho_{f f}(\tau) .
\end{aligned}
$$

In this formula one has the typical transition probability between an initial state $f, \lambda^{\prime}$ and a final state $k, \lambda$, averaged over all possible initial configurations and summed over all possible final states for the macrosystem; that is to say, contributions from both coherent and diffuse scattering are included. It might be instructive to show in a different way the connection between the last term of Eq. (2.7) and the total scattering cross section, referring to a famous paper by van Hove [11]. Taking for concreteness the Fermi pseudopotential (see next paragraph), whose Fourier transform is simply the constant $\widetilde{V}=\left(2 \pi \hbar^{2} / m\right) b$, we evaluate the diagonal element of Eq. (2.12), assuming that $u_{f}$ are given by plane waves (the indexes $f, g, h$, and $k$ becoming momenta), thus obtaining $\left[N(\boldsymbol{x})=\psi^{\dagger}(\boldsymbol{x}) \psi(\boldsymbol{x})\right]$ :

$$
\begin{aligned}
& \frac{2 \varepsilon}{\hbar}|\widetilde{V}|^{2} \sum_{\lambda, \lambda^{\prime}} \int \frac{d^{3} \boldsymbol{P}}{(2 \pi \hbar)^{3}} \int \frac{d^{3} \boldsymbol{q}}{(2 \pi \hbar)^{3}} \int d^{3} \boldsymbol{x} \int d^{3} \boldsymbol{y} e^{-(i / \hbar)\{\boldsymbol{k}-[\boldsymbol{P}+(\boldsymbol{q} / 2)]\} \cdot \boldsymbol{x}} \frac{\left\langle\lambda^{\prime}|N(\boldsymbol{x})| \lambda\right\rangle}{E_{k}+E_{\lambda^{\prime}}-\frac{1}{2 m}\left(\boldsymbol{P}+\frac{1}{2} \boldsymbol{q}\right)^{2}-E_{\lambda}-i \varepsilon}\left\langle\boldsymbol{P}+\frac{1}{2} \boldsymbol{q}|\hat{\varrho}| \boldsymbol{P}\right. \\
& \left.-\frac{1}{2} \boldsymbol{q}\right\rangle\left\langle\lambda\left|\varrho^{m}(\tau)\right| \lambda\right\rangle \frac{\left\langle\lambda|N(\boldsymbol{y})| \lambda^{\prime}\right\rangle}{E_{k}+E_{\lambda^{\prime}}-\frac{1}{2 m}\left(\boldsymbol{P}-\frac{1}{2} \boldsymbol{q}\right)^{2}-E_{\lambda}+i \varepsilon} e^{(i / \hbar)\{\boldsymbol{k}-[\boldsymbol{P}-(q / 2)]\} \cdot \boldsymbol{y}},
\end{aligned}
$$

and supposing $\hat{\varrho}$ such that the energies in the denominators may be considered approximately equal, introducing the Wigner function for the neutron,

$$
f_{w}(\boldsymbol{x}, \boldsymbol{p})=\int \frac{d^{3} \boldsymbol{q}}{(2 \pi \hbar)^{3}} e^{(i / \hbar) \boldsymbol{x} \cdot \boldsymbol{q}}\left\langle\boldsymbol{p}+\frac{1}{2} \boldsymbol{q}|\hat{\varrho}| \boldsymbol{p}-\frac{1}{2} \boldsymbol{q}\right\rangle
$$

one easily has

$$
\frac{2 \pi}{\hbar}\left(\frac{1}{2 \pi \hbar}\right)^{4}|\widetilde{V}|^{2} \int d^{3} \boldsymbol{P} \int d t \int d^{3} \boldsymbol{r} e^{-(i / \hbar)\left[\left(P^{2} / 2 m\right)-E_{k}\right] t+(i / \hbar)(\boldsymbol{P}-\boldsymbol{k}) \cdot \boldsymbol{r}} \int d^{3} \boldsymbol{X} f_{w}(\boldsymbol{X}, \boldsymbol{P})\left\langle N\left(\boldsymbol{X}-\frac{\boldsymbol{r}}{2}\right) N\left(\boldsymbol{X}+\frac{\boldsymbol{r}}{2}, t\right)\right\rangle,
$$


where $\langle\cdots\rangle \equiv \operatorname{Tr}_{\mathcal{H}_{F}}\left(\cdots \varrho^{m}\right)$, and $N(\boldsymbol{x}, t)$ denotes the operator in the Heisenberg picture. We have thus recovered the typical factorized structure appearing in the expression for the scattering cross section of a neutron off a macroscopic system: the square modulus of the Fourier transform of the interaction potential times the dynamic structure function depending on transferred momentum and energy, with the refinement that it is here weighted according to the position and momentum distribution of the incoming particle. For the nondiagonal matrix element one can expect to obtain analogous results if the quantities appearing in Eq. (2.12) are sufficiently slowly varying functions of their arguments, so that, in the continuous limit, an interpolation formula of the form

$$
\begin{aligned}
& \varepsilon \int d \xi \frac{g(\xi)}{(\alpha+\xi+i \varepsilon)(\beta+\xi-i \varepsilon)} \approx \pi \int d \xi g(\xi) \delta(\beta+\xi) \\
& \approx \pi \int d \xi g(\xi) \delta(\alpha+\xi), \quad|\alpha-\beta| \ll \varepsilon
\end{aligned}
$$

with $g(\xi)$ a suitably smooth function, may be used. The failure of such an approximation and thus the relevance of the actual value of the parameter $\varepsilon$ in the final expression might be traced back to the breakdown of the approximations that have led to the Markovian evolution generated by the master equation (2.7).

\section{OPTICAL BEHAVIOR}

We now devote our attention to the interaction of neutrons with matter. This field is well suited to test our formalism, both because of the very refined experiments that have been carried out in neutron interferometry $[1,14]$, and because of the very well-studied description of neutron optics phenomena, as developed, for example, in the book by Sears [3] that we will take as basic reference. As a first step we want to consider the coherent interaction of neutrons with matter, and therefore in Eq. (2.7) we neglect the last contribution, linked to incoherent processes. As we will see later, this term implies indeed a smaller correction in the case of neutron scattering. We are left with

$\frac{d \varrho_{k h}}{d \tau}=-\frac{i}{\hbar}\left(E_{k}-E_{h}\right) \varrho_{k h}-\frac{i}{\hbar} \sum_{f} Q_{k f} \varrho_{f h}+\frac{i}{\hbar} \sum_{g} \varrho_{k g} Q_{h g}^{*}$,

and we need a suitable expression for the operator

$$
Q_{k f}=\operatorname{Tr}_{\mathcal{H}_{F}}\left[T_{f}^{k}\left(E_{k}+i \varepsilon\right) \varrho^{m}(\tau)\right] .
$$

Following Sears, we adopt the Fermi pseudopotential to describe the neutron nucleus interaction in impulse approximation; let us recall the form of the $T$ matrix in the context of the elementary theory of dispersion,

$$
T=\frac{2 \pi \hbar^{2}}{m} \sum_{\alpha} b_{\alpha} \sum_{i=1}^{N_{\alpha}} \delta^{3}\left(\hat{\mathrm{x}}-\boldsymbol{R}_{i}\right),
$$

where $\hat{\mathrm{x}}$ is the position operator for the neutron, $\boldsymbol{R}_{i}$ the position operator for the $i$ th nucleus of type $\alpha, b_{\alpha}$ the bound scattering length, depending on isotope and spin orientation, $m$ the neutron mass, $N_{\alpha}$ the number of nuclei of type $\alpha$. An operator of form (3.2), that is to say, a sum over one-particle operators, is expressed in second quantization by

$$
T=\frac{2 \pi \hbar^{2}}{m} \sum_{\alpha} b_{\alpha} \int d^{3} \boldsymbol{x} \psi_{\alpha}^{\dagger}(\boldsymbol{x}) \delta^{3}(\hat{\mathrm{x}}-\boldsymbol{x}) \psi_{\alpha}(\boldsymbol{x}),
$$

where $\psi_{\alpha}(\boldsymbol{x})$ is the field operator, acting in the Fock space of the macrosystem, corresponding to particles of type $\alpha$. For the sake of simplicity, from now on we will consider one kind of particle, thus dropping the subscript $\alpha$. Furthermore we will assume that $b$ is a real quantity, since we are not going to deal with absorption phenomena. As we shall see in Sec. IV, we concentrate on the non-Hermiticity of the potential connected with incoherent processes and not with net absorption. A phenomenological description as given by Eq. (3.3) falls within the class of effective potentials considered in the previous paragraph, and corresponds to the following interaction kernel:

$$
t(z, x-y)=\frac{2 \pi \hbar^{2}}{m} b \delta^{3}(x-y),
$$

leading to

$$
\begin{aligned}
T_{f}^{k}\left(E_{k}+i \varepsilon\right)= & \frac{2 \pi \hbar^{2}}{m} b \int d^{3} \boldsymbol{x} \int d^{3} \boldsymbol{y} \psi^{\dagger}(\boldsymbol{x}) u_{k}^{*}(\boldsymbol{y}) \\
& \times \delta^{3}(\boldsymbol{x}-\boldsymbol{y}) u_{f}(\boldsymbol{y}) \psi(\boldsymbol{x}) .
\end{aligned}
$$

Equation (3.1) thus becomes, in operator form,

$$
\begin{aligned}
\frac{d \hat{\varrho}(\tau)}{d t}= & -\frac{i}{\hbar}\left[\hat{\mathrm{H}}_{0}, \hat{\varrho}(\tau)\right] \\
& -\frac{i}{\hbar} \frac{2 \pi \hbar^{2}}{m} b \int d^{3} \boldsymbol{x}\left\langle\psi^{\dagger}(\boldsymbol{x}) \psi(\boldsymbol{x})\right\rangle_{\tau} \delta^{3}(\hat{\mathrm{x}}-\boldsymbol{x}) \hat{\varrho}(\tau) \\
& +\frac{i}{\hbar} \frac{2 \pi \hbar^{2}}{m} b \int d^{3} \boldsymbol{x}\left\langle\psi^{\dagger}(\boldsymbol{x}) \psi(\boldsymbol{x})\right\rangle_{\tau} \hat{\varrho}(\tau) \delta^{3}(\hat{\mathrm{x}}-\boldsymbol{x}),
\end{aligned}
$$

where $\hat{\mathrm{x}}$ is the position operator for the neutron and $\langle A\rangle_{\tau} \equiv \operatorname{Tr}_{\mathcal{H}_{F}}\left(\varrho^{m}(\tau) A\right)$. If we consider only pure states and assume the macrosystem to be at equilibrium $\left(\langle\cdots\rangle_{\tau} \equiv\langle\cdots\rangle\right)$, Eq. (3.5) is equivalent to the following stationary Schrödinger equation:

$$
\left\{-\frac{\hbar^{2}}{2 m} \Delta_{x}+\frac{2 \pi \hbar^{2}}{m} b\left\langle\psi^{\dagger}(\boldsymbol{x}) \psi(\boldsymbol{x})\right\rangle\right\} \boldsymbol{\phi}(\boldsymbol{x})=E \boldsymbol{\phi}(\boldsymbol{x}),
$$

which, remembering that the average particle density $\left\langle\Sigma_{i} \delta^{3}\left(\boldsymbol{x}-\boldsymbol{R}_{i}\right)\right\rangle$ is given in second quantization by $\left\langle\psi^{\dagger}(\boldsymbol{x}) \psi(\boldsymbol{x})\right\rangle$, is exactly the equation used by Sears to describe all coherent neutron optical phenomena, here recovered in a straightforward, alternative way, though in a very different framework. The term

$$
\frac{2 \pi \hbar^{2}}{m} b\left\langle\psi^{\dagger}(\boldsymbol{x}) \psi(\boldsymbol{x})\right\rangle
$$


is called the optical potential, and assumes different expressions according to the structure of the system. If the medium can be considered homogeneous, with density $n_{o}$, Eq. (3.6) describes propagation of matter waves with an index of refraction given by

$$
n=\left(1-\frac{2 \pi \hbar^{2}}{m E} b n_{o}\right)^{1 / 2} \simeq 1-\frac{\lambda^{2}}{2 \pi} b n_{o},
$$

as first obtained by Goldberger and Seitz [13] in the absence of absorption. This is the formula currently used to calculate phase shifts in neutron interferometry experiments [1]:

$$
e^{i \chi}=e^{i(n-1)(2 \pi / \lambda) D}=e^{-i n_{o} b \lambda D},
$$

where $D$ is the thickness of the sample.

In a similar way, from Eq. (3.1) we can obtain a more general formula for the refractive index introduced for the first time by Lax [12]. Starting from the general expression (2.11), the potential term in Eq. (3.1) becomes

$$
\begin{aligned}
\sum_{f} Q_{k f} \varrho_{f h}(\tau)= & \sum_{f} \operatorname{Tr}_{\mathcal{H}_{F}} \int d^{3} \boldsymbol{x} \int d^{3} \boldsymbol{y} \psi^{\dagger}(\boldsymbol{x}) u_{k}^{*}(\boldsymbol{y}) \\
& \times t\left(E_{k}+i \boldsymbol{\varepsilon}, \boldsymbol{x}-\boldsymbol{y}\right) u_{f}(\boldsymbol{y}) \psi(\boldsymbol{x}) \varrho^{m}(\tau) \varrho_{f h}(\tau)
\end{aligned}
$$

Following Lax, we suppose that the system is homogeneous, so that

$$
\operatorname{Tr}_{\mathcal{H}_{F}}\left[\psi^{\dagger}(\boldsymbol{x}) \psi(\boldsymbol{x}) \varrho^{m}(\tau)\right]=n_{o}
$$

We have

$$
\begin{aligned}
\sum_{f} Q_{k f} \varrho_{f h}(\tau)= & n_{o} \sum_{f} \int d^{3} \boldsymbol{x} t\left(E_{k}+i \varepsilon, \boldsymbol{x}\right) \\
& \times \int d^{3} \boldsymbol{y} u_{k}^{*}(\boldsymbol{y}) u_{f}(\boldsymbol{y}) \varrho_{f h}(\tau) \\
= & n_{o} \int d^{3} \boldsymbol{x} t\left(E_{k}+i \varepsilon, \boldsymbol{x}\right) \varrho_{k h}(\tau),
\end{aligned}
$$

where we have exploited the orthogonality between the states $\left\{u_{f}\right\}$, thus obtaining the matrix element of the $T$ operator for forward scattering, averaged over the possible states of the macrosystem. Taking the relation between the $T$ operator and scattering amplitude into account, we come to

$$
-n_{o} \frac{2 \pi \hbar^{2}}{m} f\left(0, E_{k}\right) \varrho_{k h}(\tau)
$$

Inserted in the Schrödinger equation, this term is equivalent to an index of refraction of the form

$$
n=\left(1+\frac{2 \pi \hbar^{2}}{m E_{k}} n_{o} f\left(0, E_{k}\right)\right)^{1 / 2} \simeq 1+\frac{\lambda^{2}}{2 \pi} n_{o} f\left(0, E_{k}\right),
$$

simply linked to the forward scattering amplitude. An analogous result holds for electromagnetic waves propagating in a material with low density [15]. A similar treatment has been proposed [16] and adopted (see, for example, Ref. [17]) in the description of the propagation of atoms through a dilute medium, showing the interest of similar descriptions also for atom optics. In the case of thermal neutrons the scattering amplitude is isotropic within a very good approximation and is given in terms of the scattering length by the simple formula $f=-b$, which reduces Eq. (3.9) to Eq. (3.7).

So far we have shown how, starting from Eq. (2.7) and neglecting the incoherent term, we can recover some important results obtained within the framework of multiplescattering theory and used to describe the coherent interaction of neutrons with matter. Our formalism puts into evidence the statistical operator of the macrosystem, the $T$ matrix and the scattering amplitude, so that phenomenological inputs are rather direct. Further improvements of the formulas obtained are allowed by the presence of $\varrho^{m}(\tau)$ and depend on its evaluation. The correction factor $c$ that Lax includes in Eq. (3.9) to obtain the index of refraction

$$
n \simeq 1+\frac{\lambda^{2}}{2 \pi} n_{o} c f\left(0, E_{k}\right),
$$

is connected to fulfillment of the optical theorem, which, in our formalism, as we will see in Sec. IV, is related to the presence of the incoherent contribution.

\section{INCOHERENT CONTRIBUTION}

We now come to the main statement of this paper, the connection between the contributions other than the commutator in Eq. (2.10) and the dynamic structure function, together with the relevance of this relationship to the optical theorem. As observed by Sears, an expression of the form (3.7) or (3.9) for the refractive index does not include the contribution to the attenuation of the coherent wave in the medium due to diffuse scattering and, hence, violates the "'optical theorem' of scattering theory [5,3,18]. To overcome this difficulty, he refrained from ad hoc assumptions as in Ref. [19], which amount to introducing a suitable imaginary contribution to the potential, and considers a rigorous theory of dispersion. In this more accurate treatment, Eq. (3.2) is replaced by

$$
T=\frac{2 \pi \hbar^{2}}{m} \sum_{\alpha} f_{\alpha} \sum_{i=1}^{N_{\alpha}} \delta^{3}\left(\hat{\mathrm{x}}-\boldsymbol{R}_{i}\right)
$$

and $f_{\alpha}$ has the general expression ( $\boldsymbol{k}$ is the incident neutron momentum)

$$
f_{\alpha}=-b_{\alpha}+\frac{i}{\hbar} k b_{\alpha}^{2}+O\left(k^{2}\right)
$$

where the second term had been previously omitted because of its smallness, since typically $(1 / \hbar) k b \leqslant 10^{-4}$. Furthermore, the scattering amplitude is to be multiplied by a constant $c$ which should take local-field corrections into account and whose value depends only on the temperature, density, and chemical composition of the medium. Sears obtained an estimate for this constant in terms of the structure function of the macroscopic scatterer in the case of an homogeneous medium, applying a multiple wave formalism to solve the scattering problem, and drawing strong analogies to the usual descriptions of propagation of electromagnetic waves. In this 
way he recovered a correspondence between the attenuation of the coherent wave in the medium and diffuse scattering. In the following we shall set $f_{\alpha}=f \forall \alpha$ and consider only real $b$, in order to concentrate upon diffuse scattering, neglecting absorption. By diffuse scattering we intend all scattering that is not coherent in the absolute sense, that is, elastic and coherent (for the distinction between absolute and relative incoherence see for example [3,12]). To compare with these more refined results we have to consider all contributions in Eq. (2.10). Let us stress from the very beginning some general features of this expression, thanks to which it can describe more general physical situations than those arising in an evolution driven by a Schrödinger-like equation. The last two terms

$$
-\frac{1}{\hbar}\left\{\frac{1}{2} \sum_{\xi, \lambda} \hat{\mathrm{L}}_{\lambda \xi}^{\dagger} \hat{\mathrm{L}}_{\lambda \xi}, \hat{\varrho}\right\}+\frac{1}{\hbar} \sum_{\xi, \lambda} \hat{\mathrm{L}}_{\lambda \xi} \hat{\varrho} \hat{\mathrm{L}}_{\lambda \xi}^{\dagger}
$$

allow for the presence of a non-self-adjoint potential which is nevertheless not linked to real absorption. This is the case for the present treatment, in which the imaginary part of the optical potential is to be traced back to the existence of diffuse scattering, as opposed to the coherent wavelike behavior. Attenuation of the "coherent wave"' is due to the presence of the anticommutator term, responsible for the imaginary potential, balanced by the last contribution, typically incoherent in that it leads from a pure state to a mixture. This last term is given by a sum over subcollections, formally similar to the expression that we would obtain for the statistical operator after the measurement of a given observable (see Ref. [7]). The subcollections are denoted by the indexes $\lambda \xi$, which specify a change of the state of the macroscopic system, caused by interaction with the microsystem, thus making this contribution to the dynamics incoherent. In fact, we will see in the case of neutron-matter interaction that the trace of this term gives all the contributions to incoherent scattering, that is, the total diffusion cross section. The balance between the two terms of Eq. (4.1) accounts for the fulfilment of the optical theorem.

To see this let us now consider Eq. (4.1) in more detail. Starting from Eqs. (2.8) and (2.11), introducing a Laplace transform for the energy dependence of the effective $T$ matrix

$$
t(E, \boldsymbol{x})=\int_{0}^{\infty} d \sigma e^{(i / \hbar) E \sigma} \bar{t}(\sigma, \boldsymbol{x}),
$$

together with the following expression for the density number operator in terms of creation and destruction operators with specified momentum:

$$
N(\boldsymbol{x})=\psi^{\dagger}(\boldsymbol{x}) \psi(\boldsymbol{x})=\frac{1}{\mathrm{~V}} \sum_{\kappa, P} e^{-(i / \hbar) \kappa \cdot x} b_{P+(\kappa / 2)}^{\dagger} b_{P-(\kappa / 2)},
$$

we obtain

$$
\begin{aligned}
\hat{\mathrm{L}}_{\lambda \xi}= & \frac{i}{\hbar} \sqrt{2 \varepsilon \pi_{\xi}} \frac{1}{\mathrm{~V}} \sum_{\kappa, P} \int_{0}^{\infty} d \tau e^{-(\varepsilon / \hbar) \tau} \\
& \times \int_{0}^{\infty} d \sigma \int d^{3} \boldsymbol{x}^{\prime \prime} e^{-(\varepsilon / \hbar) \sigma} e^{-i / \hbar \hat{\mathrm{H}}_{0}(\tau-\sigma)} \bar{t}\left(\sigma, \boldsymbol{x}^{\prime \prime}-\hat{\mathrm{x}}\right) \\
& \times e^{(i / \hbar) \hat{\mathrm{H}}_{0} \tau} e^{-(i / \hbar) \kappa \cdot x^{\prime \prime}}\langle\lambda| e^{-(i / \hbar) H_{m} \tau} \\
& \times b_{P+(\kappa / 2)}^{\dagger} b_{P-(\kappa / 2)} e^{(i / \hbar) H_{m} \tau}|\xi\rangle
\end{aligned}
$$

where $\mathrm{V}$ is the volume of the region in which the system is supposed to be confined. Indicating by $\widetilde{t}(E, \boldsymbol{\kappa})$ the Fourier transform of the potential with respect to space

$$
\widetilde{t}(E, \boldsymbol{\kappa})=\int d^{3} \boldsymbol{x} t(E, \boldsymbol{x}) e^{-(i / \hbar) \boldsymbol{\kappa} \cdot \boldsymbol{x}},
$$

and after some simple manipulations one comes to

$$
\begin{aligned}
\hat{\mathrm{L}}_{\lambda \xi}= & \frac{i}{\hbar} \sqrt{2 \varepsilon \pi_{\xi}} \frac{1}{\mathrm{~V}} \sum_{\kappa, P} \int_{0}^{\infty} d \tau e^{-(\varepsilon / \hbar) \tau} \widetilde{t}\left(\hat{\mathrm{H}}_{0}+i \varepsilon, \boldsymbol{\kappa}\right) \\
& \times e^{(i / \hbar)\left(\boldsymbol{\kappa}^{2} / 2 m\right) \tau} e^{(i / \hbar)(\boldsymbol{\kappa} \cdot \hat{\mathrm{p}} / m) \tau} \\
& \times e^{-(i / \hbar) \boldsymbol{\kappa} \cdot \hat{\mathrm{x}}} \\
& \times\left\langle\lambda\left|e^{-(i / \hbar) H_{m} \tau} b_{P+(\kappa / 2)}^{\dagger} b_{P-(\kappa / 2)} e^{(i / \hbar) H_{m} \tau}\right| \xi\right\rangle,
\end{aligned}
$$

to be inserted into Eq. (4.1). Before doing this let us introduce the useful notation

$$
\begin{gathered}
e^{-(i / \hbar) H_{m} \tau} A e^{(i / \hbar) H_{m} \tau}=\sum_{\Delta} e^{-(i / \hbar) \Delta t}(A)_{\Delta}, \\
(A)_{\Delta}=\sum_{E}|E+\Delta\rangle\langle E+\Delta|A| E\rangle\langle E|,
\end{gathered}
$$

$$
(A)_{\Delta}^{\dagger}=\left(A^{\dagger}\right)_{-\Delta} \text {. }
$$

We have

$$
\begin{aligned}
\frac{1}{\hbar} \sum_{\xi, \lambda} \hat{\mathrm{L}}_{\lambda \xi} \hat{\varrho} \hat{\mathrm{L}}_{\lambda \xi}^{\dagger}= & \frac{2 \varepsilon}{\hbar^{3} \mathrm{~V}^{2}} \sum_{\kappa, P} \sum_{\kappa^{\prime}, P^{\prime}} \int_{0}^{\infty} d \tau e^{-(\varepsilon / \hbar) \tau} e^{(i / \hbar)\left(\boldsymbol{\kappa}^{2} / 2 m\right) \tau} e^{(i / \hbar)(\boldsymbol{\kappa} \cdot \hat{\mathrm{p}} / m) \tau} \widetilde{t}\left(\hat{\mathrm{H}}_{0}+i \varepsilon, \boldsymbol{\kappa}\right) e^{-(i / \hbar) \boldsymbol{\kappa} \cdot \hat{\mathrm{x}}} \hat{\varrho} e^{(i / \hbar) \boldsymbol{\kappa}^{\prime} \cdot \hat{\mathrm{x}}} \tilde{t} \widetilde{\mathrm{H}}^{\dagger}\left(\hat{\mathrm{H}}+i \varepsilon, \boldsymbol{\kappa}^{\prime}\right) \\
& \times \int_{0}^{\infty} d \tau^{\prime} e^{-(\varepsilon / \hbar) \tau^{\prime}} e^{-(i / \hbar)\left(\boldsymbol{\kappa}^{\prime 2} / 2 m\right) \tau^{\prime}} e^{-(i / \hbar)\left(\boldsymbol{\kappa}^{\prime} \cdot \hat{\mathrm{p}} / m\right) \tau^{\prime}} \\
& \times \sum_{\Delta, \Delta^{\prime}} e^{-(i / \hbar) \Delta \tau} \operatorname{Tr}_{\mathcal{H}_{F}\left[\left(b_{P+(\kappa / 2)}^{\dagger} b_{P-(\kappa / 2)}\right)_{\Delta} \varrho^{m}\left(b_{P^{\prime}-\left(\kappa^{\prime} / 2\right)}^{\dagger} b_{P^{\prime}+\left(\kappa^{\prime} / 2\right)}\right)_{-\Delta^{\prime}}\right] e^{i / \hbar \Delta^{\prime} \tau^{\prime}}}
\end{aligned}
$$


and similarly for the anticommutator part. An important simplification takes place if one can use symmetry under time and space translations. Time translation invariance occurs if, at least with reference to the interaction with the microsystem, matter can be considered at equilibrium; then

$$
\operatorname{Tr}_{\mathcal{H}_{F}}\left(A_{\Delta} \varrho^{m} B_{-\Delta^{\prime}}\right)=\delta_{\Delta, \Delta^{\prime}} \operatorname{Tr}_{\mathcal{H}_{F}}\left(A_{\Delta} \varrho^{m} B_{-\Delta}\right)
$$

Similarly, space translation invariance implies

$$
\begin{aligned}
\operatorname{Tr}_{\mathcal{H}_{F}}\left[\left(b_{P+(\kappa / 2)}^{\dagger} b_{P-(\kappa / 2)}\right)_{\Delta} \varrho^{m}\left(b_{P^{\prime}-\left(\kappa^{\prime} / 2\right)}^{\dagger} b_{P^{\prime}+\left(\kappa^{\prime} / 2\right)}\right)_{-\Delta^{\prime}}\right] \\
=\delta_{\Delta, \Delta^{\prime}} \delta_{\kappa, \kappa^{\prime}} \operatorname{Tr}_{\mathcal{H}_{F}}\left[\left(b_{P+(\kappa / 2)}^{\dagger} b_{P-(\kappa / 2)}\right)_{\Delta} \varrho^{m}\right. \\
\left.\quad \times\left(b_{P^{\prime}-(\kappa / 2)}^{\dagger} b_{P^{\prime}+(\kappa / 2)}\right)_{-\Delta}\right]
\end{aligned}
$$

such a symmetry can be implemented at equilibrium in the thermodynamic limit, and can be practically assumed for a microsystem interacting with a homogeneous portion of a macrosystem. Then one has, also the $\tau, \tau^{\prime}$ integrals performing,

$$
\begin{aligned}
& -\frac{1}{\hbar}\left\{\frac{1}{2} \sum_{\xi, \lambda} \hat{\mathrm{L}}_{\lambda \xi}^{\dagger} \hat{\mathrm{L}}_{\lambda \xi}, \hat{\varrho}\right\}+\frac{1}{\hbar} \sum_{\xi, \lambda} \hat{\mathrm{L}}_{\lambda \xi} \hat{\varrho} \hat{\mathrm{L}}_{\lambda \xi}^{\dagger} \\
& =-\frac{\varepsilon}{\hbar \mathrm{V}^{2}} \sum_{\kappa, \Delta}\left(\left\{\hat{\varrho}, e^{(i / \hbar) \boldsymbol{\kappa} \cdot \hat{\mathrm{x}}} \frac{1}{\frac{\boldsymbol{\kappa} \cdot \hat{\mathrm{p}}}{m}+\frac{\boldsymbol{\kappa}^{2}}{2 m}-\Delta-i \varepsilon}\right.\right. \\
& \times \widetilde{t}^{\dagger}\left(\hat{\mathrm{H}}_{0}+i \varepsilon, \boldsymbol{\kappa}\right) \widetilde{t}\left(\hat{\mathrm{H}}_{0}+i \varepsilon, \boldsymbol{\kappa}\right) \\
& \left.\times \frac{1}{\frac{\boldsymbol{\kappa} \cdot \hat{\mathrm{p}}}{m}+\frac{\boldsymbol{\kappa}^{2}}{2 m}-\Delta+i \varepsilon} e^{-(i / \hbar) \boldsymbol{\kappa} \cdot \hat{\mathrm{x}}}\right\} \\
& -2 \frac{1}{\frac{\boldsymbol{\kappa} \cdot \hat{\mathrm{p}}}{m}+\frac{\boldsymbol{\kappa}^{2}}{2 m}-\Delta+i \varepsilon} \widetilde{t}\left(\hat{\mathrm{H}}_{0}+i \varepsilon, \boldsymbol{\kappa}\right) e^{-(i / \hbar) \boldsymbol{\kappa} \cdot \hat{\mathrm{x}}} \\
& \left.\times \hat{\varrho} e^{(i / \hbar) \boldsymbol{\kappa} \cdot \hat{\mathrm{x}}} \widetilde{t}^{\dagger}\left(\hat{\mathrm{H}}_{0}+i \varepsilon, \boldsymbol{\kappa}\right) \frac{1}{\frac{\boldsymbol{\kappa} \cdot \hat{\mathrm{p}}}{m}+\frac{\boldsymbol{\kappa}^{2}}{2 m}-\Delta-i \varepsilon}\right) \\
& \times \varrho^{M}(\kappa, \Delta),
\end{aligned}
$$

where

$$
\begin{aligned}
\varrho^{M}(\kappa, \Delta) \equiv & \operatorname{Tr}_{\mathcal{H}_{F}}\left[\left(\sum_{P} b_{P+(\kappa / 2)}^{\dagger} b_{P-(\kappa / 2)}\right)_{\Delta}\right. \\
& \left.\times \varrho^{m}\left(\sum_{P} b_{P-(\kappa / 2)}^{\dagger} b_{P+(\kappa / 2)}\right)_{-\Delta}\right],
\end{aligned}
$$

or, equivalently, introducing the $\hat{\mathrm{x}}, \hat{\mathrm{p}}$-dependent amplitude

$$
\check{t}\left(\hat{\mathrm{H}}_{0}+i \varepsilon, \boldsymbol{\kappa}, \hat{\mathrm{x}}\right)=e^{(i / \hbar) \boldsymbol{\kappa} \cdot \hat{\mathrm{x}}_{t}}\left(\hat{\mathrm{H}}_{0}+i \varepsilon, \boldsymbol{\kappa}\right) e^{-(i / \hbar) \boldsymbol{\kappa} \cdot \hat{\mathrm{x}}}
$$

in the form

$$
\begin{aligned}
-\frac{1}{\hbar \mathrm{V}^{2}} & \sum_{\kappa, \Delta}\left(\left\{\hat{\varrho}, \check{t}^{\dagger}\left(\hat{\mathrm{H}}_{0}+i \varepsilon, \boldsymbol{\kappa}, \hat{\mathrm{x}}\right) \frac{\varepsilon}{\left(\frac{\boldsymbol{\kappa} \cdot \hat{\mathrm{p}}}{m}-\frac{\boldsymbol{\kappa}^{2}}{2 m}-\Delta\right)^{2}+\varepsilon^{2}}\right.\right. \\
& \times \check{t}\left(\hat{\mathrm{H}}_{0}+i \varepsilon, \boldsymbol{\kappa}, \hat{\mathrm{x}}\right)-2 \varepsilon e^{-(i / \hbar) \boldsymbol{\kappa} \cdot \hat{\mathrm{x}}^{2}\left(\hat{\mathrm{H}}_{0}+i \varepsilon, \boldsymbol{\kappa}, \hat{\mathrm{x}}\right)} \\
& \times \frac{1}{\boldsymbol{\kappa} \cdot \hat{\mathrm{p}}} \frac{\boldsymbol{\kappa}^{2}}{m}-\Delta+i \varepsilon \frac{1}{\frac{\boldsymbol{\kappa} \cdot \hat{\mathrm{p}}}{m}-\frac{\boldsymbol{\kappa}^{2}}{2 m}-\Delta-i \varepsilon} \\
& \times \check{t}^{\dagger}\left(\hat{\mathrm{H}}_{0}+i \varepsilon, \boldsymbol{\kappa}, \hat{\mathrm{x}}\right) e^{(i / \hbar) \boldsymbol{\kappa} \cdot \hat{\mathrm{x}} \varrho^{M}(\boldsymbol{\kappa}, \Delta)}
\end{aligned}
$$

Introducing this explicit representation in Eq. (2.10), one obtains the typical master equation of Brownian motion, that can be further simplified in the assumption of small momentum transfer, i.e., expanding the expression with respect to $\boldsymbol{\kappa} \cdot \hat{\mathrm{x}}$ and $\boldsymbol{\kappa} \cdot \hat{\mathrm{p}}$. Exploiting the fact that $\varrho^{M}(0, \Delta)$ contains a $\delta_{\Delta, 0}$ factor, one can immediately see by inspection that the $\kappa=0$ contributions cancel each other, provided the effective $T$ matrix is a slow function of energy,

$$
\begin{aligned}
\langle\boldsymbol{k}|\hat{\varrho}| \boldsymbol{f}\rangle \widetilde{t}\left(E_{k}, 0\right) \widetilde{t}^{\dagger}\left(E_{f}, 0\right) \approx & \langle\boldsymbol{k}|\hat{\varrho}| \boldsymbol{f}\rangle \frac{1}{2}\left[\widetilde{t}^{\dagger}\left(E_{k}, 0\right) \widetilde{t}\left(E_{k}, 0\right)\right. \\
& \left.+\widetilde{t}^{\dagger}\left(E_{f}, 0\right) \widetilde{t}\left(E_{f}, 0\right)\right] ;
\end{aligned}
$$

on the other hand, for a homogeneous medium the $\kappa=0$ contributions are equal to those obtained by writing the correlation function as a factorized product

$$
\begin{aligned}
\operatorname{Tr}_{\mathcal{H}_{F}} & {\left[\left(\sum_{P} b_{P+(\kappa / 2)}^{\dagger} b_{P-(\kappa / 2)}\right)_{\Delta} \varrho^{m}\left(\sum_{P} b_{P-(\kappa / 2)}^{\dagger} b_{P+(\kappa / 2)}\right)_{-\Delta}\right] } \\
& \rightarrow \operatorname{Tr}_{\mathcal{H}_{F}}\left[\left(\sum_{P} b_{P+(\kappa / 2)}^{\dagger} b_{P-(\kappa / 2)}\right)_{\Delta} \varrho^{m}\right] \operatorname{Tr}_{\mathcal{H}_{F}}\left[\left(\sum_{P} b_{P-(\kappa / 2)}^{\dagger} b_{P+(\kappa / 2)}\right)_{-\Delta} \varrho^{m}\right],
\end{aligned}
$$

provided we assume the condition of "normal density fluctuations,", $\left(\left\langle N^{2}\right\rangle-\langle N\rangle^{2}\right) / V^{2} \ll n_{o}^{2}$. Instead of restricting the sum to the $\kappa \neq 0$ contributions, we can therefore subtract from the correlation function its factorized part. After straightforward manipulations, using

$$
\sum_{P} b_{P+(\kappa / 2)}^{\dagger} b_{P-(\kappa / 2)}=\int d^{3} \boldsymbol{x} \psi^{\dagger}(\boldsymbol{x}) \psi(\boldsymbol{x}) e^{(i / \hbar) \boldsymbol{\kappa} \cdot \boldsymbol{x}}, \quad \operatorname{Tr}_{\mathcal{H}_{F}}\left[(A)_{\Delta} \varrho^{m}(B)_{-\Delta}\right]=\int \frac{d t}{2 \pi \hbar} e^{-(i / \hbar) \Delta t}\langle B A(t)\rangle,
$$

we come to 


$$
\begin{aligned}
\operatorname{Tr}_{\mathcal{H}_{F}} & {\left[\left(\sum_{P} b_{P+(\kappa / 2)}^{\dagger} b_{P-(\kappa / 2)}\right)_{\Delta} \varrho^{m}\left(\sum_{P} b_{P-(\kappa / 2)}^{\dagger} b_{P+(\kappa / 2)}\right)_{-\Delta}\right]-\operatorname{Tr}_{\mathcal{H}_{F}}\left[\left(\sum_{P} b_{P+(\kappa / 2)}^{\dagger} b_{P-(\kappa / 2)}\right)_{\Delta} \varrho^{m}\right] } \\
& \times \operatorname{Tr}_{\mathcal{H}_{F}}\left[\left(\sum_{P} b_{P-(\kappa / 2)}^{\dagger} b_{P+(\kappa / 2)}\right) \varrho_{-\Delta}^{m}\right] \\
= & \int \frac{d t}{2 \pi \hbar} e^{-(i / \hbar) \Delta t} \int d^{3} \boldsymbol{x} \int d^{3} \boldsymbol{y} e^{(i / \hbar) \boldsymbol{\kappa} \cdot(\boldsymbol{x}-\boldsymbol{y})}\langle\delta N(\boldsymbol{y}) \delta N(\boldsymbol{x}, t)\rangle,
\end{aligned}
$$

where

$$
\langle N(\boldsymbol{x})\rangle=\operatorname{Tr}_{\mathcal{H}_{F}}\left[N(\boldsymbol{x}) \varrho^{m}\right], \quad \delta N(\boldsymbol{x})=N(\boldsymbol{x})-\langle N(\boldsymbol{x})\rangle,
$$

and, finally,

$$
\begin{aligned}
-\frac{1}{\hbar}\left\{\frac{1}{2} \sum_{\xi, \lambda} \hat{\mathrm{L}}_{\lambda \xi}^{\dagger} \hat{\mathrm{L}}_{\lambda \xi}, \hat{\varrho}\right\}+\frac{1}{\hbar} \sum_{\xi, \lambda} \hat{\mathrm{L}}_{\lambda \xi} \hat{\varrho} \hat{\mathrm{L}}_{\lambda \xi}^{\dagger}= & -\frac{\varepsilon}{\hbar \mathrm{V}^{2}} \sum_{\boldsymbol{\kappa}, \Delta}\left(\left\{\hat{\varrho}, e^{(i / \hbar) \boldsymbol{\kappa} \cdot \hat{x}} \frac{1}{\frac{\boldsymbol{\kappa} \cdot \hat{\mathrm{p}}}{m}+\frac{\boldsymbol{\kappa}^{2}}{2 m}-\Delta-i \varepsilon} \widetilde{t}^{\dagger}\left(\hat{\mathrm{H}}_{0}+i \varepsilon, \boldsymbol{\kappa}\right) \widetilde{t}\left(\hat{\mathrm{H}}_{0}+i \varepsilon, \boldsymbol{\kappa}\right)\right.\right. \\
& \left.\left.\times \frac{1}{\frac{\boldsymbol{\kappa} \cdot \hat{\mathrm{p}}}{m}+\frac{\boldsymbol{\kappa}^{2}}{2 m}-\Delta+i \varepsilon} e^{-(i / \hbar) \boldsymbol{\kappa} \cdot \hat{\mathrm{x}}}\right\}-2 \frac{1}{\frac{\boldsymbol{\kappa} \cdot \hat{\mathrm{p}}}{m}+\frac{\boldsymbol{\kappa}^{2}}{2 m}-\Delta+i \varepsilon} \hat{\mathrm{H}}_{0}+i \varepsilon, \boldsymbol{\kappa}\right) \\
& \left.\times e^{-(i / \hbar) \boldsymbol{\kappa} \cdot \hat{\mathrm{x}}} \hat{\varrho} e^{(i / \hbar) \boldsymbol{\kappa} \cdot \hat{x}} \widetilde{t}^{\dagger}\left(\hat{\mathrm{H}}_{0}+i \varepsilon, \boldsymbol{\kappa}\right) \frac{1}{\frac{\boldsymbol{\kappa} \cdot \hat{\mathrm{p}}}{m}+\frac{\boldsymbol{\kappa}^{2}}{2 m}-\Delta-i \varepsilon}\right) \\
& \times \int \frac{d t}{2 \pi \hbar} e^{-(i / \hbar) \Delta t \int d^{3} \boldsymbol{x} \int d^{3} \boldsymbol{y} e^{(i / \hbar) \boldsymbol{\kappa} \cdot \boldsymbol{x}}\langle\delta N(\boldsymbol{y}) \delta N(\boldsymbol{x}+\boldsymbol{y}, t)\rangle .}
\end{aligned}
$$

Thanks to the last term of Eq. (2.10), it is possible to take into account collisions that modify the state of the macroscopic system (see Ref. [7]). The probability per unit time of such collisions is given by the trace of $1 / \hbar \Sigma_{\xi, \lambda} \hat{L}_{\lambda \xi} \hat{\varrho} \hat{L}_{\lambda \xi}^{\dagger}$, as seen in Sec. II. In the case considered this trace may be written as

$\frac{2 \pi}{\hbar} \frac{n_{o}}{(2 \pi \hbar)^{4}} \int d^{3} \boldsymbol{k} \int d^{3} \boldsymbol{\kappa}\langle\boldsymbol{\kappa}|\hat{\varrho}| \boldsymbol{\kappa}\rangle\left|\widetilde{t}\left(E_{k}, \boldsymbol{\kappa}-\boldsymbol{k}\right)\right|^{2} \int d t \int d^{3} \boldsymbol{x} e^{-(i / \hbar)\left[\left(\boldsymbol{\kappa}^{2} / 2 m\right)-\left(\boldsymbol{k}^{2} / 2 m\right)\right] t+(i / \hbar)(\boldsymbol{\kappa}-\boldsymbol{k}) \cdot \boldsymbol{x}} \int d^{3} \boldsymbol{y} \frac{1}{N}\langle\delta N(\boldsymbol{y}) \delta N(\boldsymbol{x}+\boldsymbol{y}, t)\rangle$,

thus again recovering the van Hove structure for the scattering cross section [compare Eq. (2.13)], with the difference that now the system is considered to be homogeneous, so that only the momentum distribution of the incoming microsystem is of relevance. Let us observe that subtraction of the uncorrelated part of the response function accounts for the fact that only diffuse scattering, that is, scattering that does not leave the macroscopic system unchanged [3], contributes to this term. We now specialize to the case of neutrons, adopting the Fermi pseudopotential given by Eq. (3.4), so that Eq. (4.5) becomes

$$
\frac{1}{\hbar} n_{o} \frac{b^{2}}{m^{2}} \int d^{3} \boldsymbol{k} \int d^{3} \boldsymbol{\kappa}\langle\boldsymbol{\kappa}|\hat{\varrho}| \boldsymbol{\kappa}\rangle S_{\mathrm{c}}\left(\frac{1}{\hbar}[\boldsymbol{\kappa}-\boldsymbol{k}], \frac{1}{\hbar}\left[\frac{\boldsymbol{\kappa}^{2}}{2 m}-\frac{\boldsymbol{k}^{2}}{2 m}\right]\right)
$$

where, denoting by $\omega$ and $\boldsymbol{q}$ energy and momentum transfer, respectively,

$$
S_{\mathrm{c}}(\boldsymbol{q}, \omega)=\frac{1}{2 \pi N} \int d t \int d^{3} \boldsymbol{x} e^{-i(\omega t-\boldsymbol{q} \cdot \boldsymbol{x})} \int d^{3} \boldsymbol{y}\langle\delta N(\boldsymbol{y}) \delta N(\boldsymbol{x}+\boldsymbol{y}, t)\rangle
$$


If the momentum distribution of the incoming particle is suitably peaked around $\boldsymbol{p}_{0}$ with respect to the momentum dependence of $S_{c}$, we have, from Eq. (4.6),

$$
\begin{gathered}
\frac{n_{o} b^{2}}{\hbar m^{2}} \int d^{3} \boldsymbol{k} \int d^{3} \boldsymbol{\kappa}\langle\boldsymbol{\kappa}|\hat{\varrho}| \boldsymbol{\kappa}\rangle S_{c}\left(\frac{1}{\hbar}\left[\boldsymbol{p}_{0}-\boldsymbol{k}\right], \omega_{p_{0}}-\omega_{k}\right) \\
=\frac{n_{o} b^{2}}{\hbar m^{2}} \int d^{3} \boldsymbol{k} S_{c}\left(\frac{1}{\hbar}\left[\boldsymbol{p}_{0}-\boldsymbol{k}\right], \omega_{p_{0}}-\omega_{k}\right),
\end{gathered}
$$

in particular, in the static limit, expression (4.6) becomes

$$
n_{o} b^{2} \frac{p_{0}}{m} \int d \Omega_{q} S_{c}(\boldsymbol{q})=n_{o} \frac{p_{0}}{m} \sigma_{d},
$$

where

$$
S_{c}(\boldsymbol{q})=\frac{1}{N} \int d^{3} \boldsymbol{x} e^{i \boldsymbol{q} \cdot \boldsymbol{x}} \int d^{3} \boldsymbol{y}\langle\delta N(\boldsymbol{y}) \delta N(\boldsymbol{x}+\boldsymbol{y})\rangle,
$$

and we have denoted by $\boldsymbol{q}$ the momentum transfer and by $\sigma_{\mathrm{d}}$ the total diffusion cross section per particle. This is the result derived by Sears for the attenuation of the coherent beam due to incoherent scattering, which he obtained by an evaluation of the local-field effects, neglected in the equation giving the optical neutron dynamics (3.6) (see Refs. [3,5,18]). In our approach, however, the incoherent contribution is already present in the equation giving the dynamics of the microsystem, being connected to the thermodynamic properties of the macrosystem through the response function $S_{c}(\boldsymbol{q}, \omega)$. This new feature is obtained by means of the more general formalism adopted, leading to a master equation of the Lindblad type for the statistical operator, in which due to the optical theorem a close correlation exists between the incoherent contribution and the imaginary part of the optical potential which is not connected to absorption. To see this correction to the optical potential let us exploit the simple relation

$$
\begin{gathered}
\hat{\mathrm{A}}=\hat{\mathrm{A}}^{\dagger}, \quad \hat{\mathrm{B}}=\hat{\mathrm{B}}^{\dagger}, \\
\hat{\mathrm{U}}=\hat{\mathrm{A}}+i \hat{\mathrm{B}} \Rightarrow \hat{\mathrm{U}} \hat{\varrho}-\hat{\varrho} \hat{\mathrm{U}}^{\dagger}=[\hat{\mathrm{A}}, \hat{\varrho}]+i\{\hat{\mathrm{B}}, \hat{\varrho}\}
\end{gathered}
$$

and write the commutator and anticommutator term of Eq. (2.10) in the form $-(i / \hbar)\left(\hat{U} \hat{\varrho}-\hat{\varrho} \hat{U}^{\dagger}\right)$. The calculation of $\hat{U}$ is essentially given by the anticommutator on the right-hand side of Eq. (4.4) and the commutator in Eq. (3.5). In the case of the Fermi pseudopotential, using Eq. (4.7), one has

$$
\begin{aligned}
\hat{U}= & \frac{2 \pi \hbar^{2}}{m} n_{o}\left[b-i \frac{b^{2}}{4 \pi} \int d^{3} \boldsymbol{k}|\boldsymbol{k}\rangle\langle\boldsymbol{k}|\right. \\
& \left.\times \int d \omega_{\kappa} \int d \Omega_{\kappa} \frac{\kappa}{\hbar} S_{c}\left(\frac{1}{\hbar}[\boldsymbol{\kappa}-\boldsymbol{k}], \frac{1}{\hbar}\left[\frac{\boldsymbol{\kappa}^{2}}{2 m}-\frac{\boldsymbol{k}^{2}}{2 m}\right]\right)\right],
\end{aligned}
$$

or, in the static limit,

$$
\hat{\mathrm{U}}=\frac{2 \pi \hbar^{2}}{m} n_{o}\left[b-i \frac{b^{2}}{4 \pi} \int d^{3} \boldsymbol{k}|\boldsymbol{k}\rangle\langle\boldsymbol{k}| \frac{k}{\hbar} \int d \Omega_{q} S_{c}(\boldsymbol{q})\right],
$$

where $\boldsymbol{q}$ denotes as usual the momentum transfer. Neglecting diffuse scattering we would have $\hat{\mathrm{U}}=\left(2 \pi \hbar^{2} / m\right) n_{o} b$, simply a $c$ number giving the usual refractive index; the remaining part is, in a sense, induced by the optical theorem. To compare with the results derived by Sears we have to consider the expression obtained for the static limit (4.9) applied to a plane wave of momentum $\boldsymbol{p}_{0}$, which gives an idealized description of the preparation of the incoming microsystem, thus leading to

$$
\hat{\mathrm{U}}=\frac{2 \pi \hbar^{2}}{m} n_{o}\left[b-i \frac{b^{2}}{4 \pi} \frac{p_{0}}{\hbar} \int d \Omega_{q} S_{c}(\boldsymbol{q})\right]
$$

this expression agrees with the results obtained relying on the idea of local-field corrections (see Ref. [3], Chap. 4); however, here Eq. (4.9) is a direct consequence of the equation driving the dynamics and of ansatz (2.11). The analysis that we put forward relies on the assumption that the main contribution to the dynamics is given by the commutator term in Eq. (2.10), while the terms in Eq. (4.1) may, as a first approximation, be neglected. This leads to an optical description, as for the case of neutrons, in which, considering the dimensionless parameter $\left(2 \pi \hbar^{2} / m E\right) n_{o} b$, the terms other than the commutator are of second order. The opposite situation takes place if the interaction is such that the main contribution is given by Eq. (4.1), while the commutator may be neglected. This happens when dissipative effects are predominant, as in the case of Brownian motion mentioned below Eq. (4.3), where incoherent interactions through collisions involving energy and momentum transfer play the main role, a case we intend to deal with in a future paper.

\section{EXPERIMENTAL IMPLICATIONS}

We now address our attention to potential experimental implications of the above-introduced description of neutronmatter interaction. Of course possible new features in the dynamics are linked to the presence of the last two terms on the right-hand-side of Eq. (2.10), as given by Eq. (4.4), and such corrections will be generally small, being of second order in $\left(2 \pi \hbar^{2} / m E\right) n_{o} b$ [typically $\left(2 \pi \hbar^{2} / m E\right) n_{o} b \leqslant 10^{-5}$ at thermal neutron energies]. In this respect interferometric experiments, in which the experimental setup is conceived in order to enhance the coherent behavior, should be particularly relevant: think, for example, of the beautiful experiments realized by the Rauch group in Wien exploiting the perfect crystal neutron interferometer $[1,2,14]$.

Now consider Eq. (2.10): the map on the right-hand side is affine and trace preserving, and therefore clearly predicts neutron conservation. Nevertheless the last contribution which offsets the anticommutator term is linked to diffuse scattering: one has neutron conservation if diffuse particles also contribute to the experimental observation. This is not so for interferometric experiments. In such cases only the wavelike behavior affects the observed dynamics, and thus only the commutator part of the evolution map is of relevance: the net result is an imaginary correction to the coherent scattering length as in Eq. (4.10), that is to say a reduction of the neutron flux responsible for the interference pattern. This fact is usually taken into account by adding an imaginary part proportional to the total scattering cross sec- 
tion $\sigma_{\mathrm{t}}$ to the phase shift calculated as in Eq. (3.8), thus including both absorption and diffuse scattering (see Refs. $[2,20])$ according to the formulas

$$
\begin{gathered}
\chi=\chi^{\prime}+i \chi^{\prime \prime}=-n_{o} b \lambda D+i n_{o} \sigma_{\mathrm{t}} \frac{D}{2}, \\
\exp (i \chi)=\exp \left(-i n_{o} b \lambda D-n_{o} \sigma_{\mathrm{t}} \frac{D}{2}\right) .
\end{gathered}
$$

In the absence of absorption this correction is considered negligible, and the relevant incident flux is often evaluated simply closing one of the two beam paths. This attitude is, however, at least in principle incorrect, as it appears taking the whole dynamics as given by Eq. (2.10) into account. In fact, when one closes the path without the sample, diffuse neutrons, which are lost for the interference pattern, having their path "labeled"' by scattering with the sample, may also contribute to the transmitted intensity. The experimental device no longer acts as an interferometer, and therefore cannot select only those neutrons that have undergone coherent interactions. This additional contribution to the transmitted neutron flux is given by the trace of the last term of Eq. (2.10), that is to say by Eq. (4.6). In calculating the amplitude of the interference pattern one should therefore rely not simply on the measured transmitted flux, but on this quantity minus the additional incoherent contribution given by Eq. (4.6), thus obtaining a reduction of this amplitude: the purely "optical" treatment leads in principle to an overestimate of the visibility of the interference pattern. This is normally not the case in real experiments, since the angle of acceptance of diffuse neutrons is very small, as for the perfect crystal neutron interferometer. Let us give some quantitative estimates of the aforementioned effect.

In order to evaluate Eq. (4.6) we have to make a definite choice for the structure function $S_{c}(\boldsymbol{q}, \omega)$, in fact Eq. (4.6) is given by

$$
\begin{aligned}
\mathcal{A} \equiv & \frac{1}{\hbar} \operatorname{Tr}_{\mathcal{H}^{(1)}} \sum_{\xi, \lambda} \hat{\mathrm{L}}_{\lambda \xi} \hat{\varrho} \hat{\varrho} \hat{\mathrm{L}}_{\lambda \xi}^{\dagger} \\
= & \frac{n_{o} b^{2}}{\hbar m^{2}} \int d^{3} \boldsymbol{k} \int d^{3} \boldsymbol{\kappa}\langle\boldsymbol{\kappa}|\hat{\varrho}| \boldsymbol{\kappa}\rangle \\
& \times S_{c}\left(\frac{1}{\hbar}[\boldsymbol{\kappa}-\boldsymbol{k}], \frac{1}{\hbar}\left[\frac{\boldsymbol{\kappa}^{2}}{2 m}-\frac{\boldsymbol{k}^{2}}{2 m}\right]\right),
\end{aligned}
$$

where the quantity $\mathcal{A}$ takes diffusion at any angle into account. In the static approximation, for a homogeneous and isotropic medium, such as a liquid or a gas, one has [3]

$$
\begin{gathered}
S_{c}(\boldsymbol{q}, \omega)=S_{c}(\boldsymbol{q}) \delta(\omega), \\
S_{c}(\boldsymbol{q})=1+n_{o} \int d^{3} \boldsymbol{r} e^{i \boldsymbol{q} \cdot \boldsymbol{r}}[g(r)-1],
\end{gathered}
$$

where $g(r)$ is the pair-correlation function. A possible choice for $g(r)$, allowing $S_{c}(\boldsymbol{q})$ to be evaluated analitically, is the following, valid for a dilute hard sphere gas with atomic diameter $a$ :

$$
g(r)= \begin{cases}0, & r<a \\ 1, & r>a .\end{cases}
$$

The quantity of interest for us is $\mathcal{A}$ in its dependence from the maximal angular acceptance $\varphi$, determined by the experimental apparatus, multiplied by the time the neutron takes to go through the sample. Supposing the momentum distribution of the incoming particle is sufficiently well peaked around $\boldsymbol{p}_{0}$, we rewrite $\mathcal{A}$ introducing the expression given by Eq. (5.1) and multiplying by the time interval, thus coming to

$$
\begin{aligned}
\mathcal{A}(\varphi)= & 2 \pi n_{o} b^{2} D \int_{0}^{\varphi} d \theta \sin \theta\left\{1-\frac{2 \pi n_{o} a^{3}}{(1-\cos \theta)}\left(\frac{\hbar}{a p_{0}}\right)^{2}\right. \\
& \times\left[\frac{\sin \left(\frac{a p_{o}}{\hbar} \sqrt{2(1-\cos \theta)}\right)}{\frac{a p_{o}}{\hbar} \sqrt{2(1-\cos \theta)}}\right. \\
& \left.-\cos \left(\frac{a p_{o}}{\hbar} \sqrt{2(1-\cos \theta)}\right)\right]
\end{aligned}
$$

where $\cos \theta=\left(\boldsymbol{p}_{0} \cdot \boldsymbol{k}\right) / p_{0}^{2}$. The primitive of this integral can be straightforwardly evaluated by a change of variables, and exploiting the fact that in our model $S_{c}(0)=1-\frac{4}{3} \pi a^{3} n_{o}$, we have an explicit representation of diffuse scattering at any angle $\varphi$ :

$$
\begin{aligned}
\mathcal{A}(\varphi)=2 \pi n_{o} b^{2} D\left\{(1-\cos \varphi)+3\left[1-S_{c}(0)\right]\right. \\
\\
\times\left(\frac{\hbar}{a p_{0}}\right)^{2}\left[\frac{\sin \left(\frac{a p_{o}}{\hbar} \sqrt{2(1-\cos \varphi)}\right)}{\left.\left.\frac{a p_{o}}{\hbar} \sqrt{2(1-\cos \varphi)}-1\right]\right\} ;}\right.
\end{aligned}
$$

considering in particular small $\varphi$, the expression may be approximated as

$$
\begin{aligned}
\mathcal{A}(\varphi) \simeq & \pi n_{o} b^{2} D\left\{\varphi^{2} S_{c}(0)+\varphi^{4}\left[\frac{1}{20}\left[1-S_{c}(0)\right]\left(\frac{a p_{0}}{\hbar}\right)^{2}\right.\right. \\
& \left.\left.-\frac{1}{12} S_{c}(0)\right]+O\left(\varphi^{6}\right)\right\} .
\end{aligned}
$$

Let us now consider the experiments performed using the perfect crystal interferometer. The angular acceptance is very small, only a few microradians for thermal neutrons [21]. Taking, for instance, a gaseous sample, an order-ofmagnitude estimate gives $\mathcal{A}(\varphi) \simeq 10^{-14}$, that is to say an extremely small quantity, in agreement with the accuracy obtained using this interferometer based on Bragg diffrac- 
tion. An interferometer based on a different physical principle could possibly lead to a higher angular acceptance, thus enhancing this effect connected to diffusion. In view of the next equation (5.2), a completely different situation arises if one considers systems with abnormally large density fluctuations, as would be the case near a first-order phase-transition.

Another point of interest is the linear dependence on $S_{c}(0)$ of the leading term in $\mathcal{A}(\varphi)$. The quantity $S_{c}(0)$ is particularly relevant from the physical point of view, being connected to the isothermal compressibility $\chi_{T}$ and to the fluctuations in the number of particles in the sample [22]:

$$
S_{c}(0)=n_{o} k_{B} T \chi_{T}=\frac{\overline{(\Delta N)^{2}}}{N} .
$$

The actual value of $S_{c}(0)$ cannot be measured experimentally from scattering experiments, and has to be obtained by an analytical continuation. The analysis we propose could provide an independent way to measure $S_{c}$ at $\boldsymbol{q}=0$. In fact in the static approximation, independently of the particular form of $S_{c}(\boldsymbol{q})$, for very small $|\boldsymbol{q}|$, that is to say for very small $\varphi$, one has, in a good approximation,

$$
\mathcal{A}(\varphi) \simeq \pi n_{o} b^{2} D S_{c}(0) \varphi^{2} .
$$

The value of $S_{c}(0)$ could then be obtained, at least in principle, comparing the amplitude of the interference pattern with the measured transmitted intensity.

\section{SUMMARY AND OUTLOOK}

The example of neutron interaction with matter has been discussed inside the approach outlined in Refs. [7-9] to describe the subdynamics of a microsystem interacting with a system having many degrees of freedom. The formal scheme leads to a generator for the irreversible time evolution of the Lindblad form, whose expression relies on suitable choices for the potential term related to the $T$ matrix and the statistical operator describing the thermodynamic state of the system. In the example considered the main ingredient is given by the Fermi pseudopotential adopted to describe the neutron-nucleus interaction in impulse approximation. Then from Eq. (2.7) we obtain, neglecting the incoherent contribution, the equation used by Sears to describe all neutron optical phenomena, as well as known expressions for the index of refraction. The incoherent contribution is necessary to fulfill the optical theorem and take diffuse scattering, that attenuates the coherent beam, into account. We have also shown how it may be connected to properties of the macrosystem, as expressed by the dynamic structure function. Furthermore possible experimental implications were discussed in Sec. V.

Even though it introduces a smaller correction the incoherent contribution is very important from the theoretical point of view. We expect that it will help in studying the tricky borderline between a pure optical wavelike behavior and the fully incoherent particlelike one, based on a diffusion equation: in fact, Eq. (2.10) leads in a direct way to the theory of Brownian motion, as stressed under Eq. (4.3); a somewhat similar treatment, in the case of an ideal gas, was given in Ref. [23]. It is not surprising that the incoherent contribution to the dynamics has grown out of a thoroughly quantum-mechanical treatment, as shown by the typical quantum-structure of the Lindblad equation, relying on noncommutating operators, in which an essential role is played by the statistical operator $Q$, rather then by the wave function $\psi$. This point is of central relevance, since the terms which describe the incoherent dynamics cannot be introduced in the formalism of the wave function, and are therefore unavoidably absent in an optical-like treatment, simply reminiscent of classical optical descriptions.

We hope that this study of the emergence of incoherence in neutron-matter interaction will lead to a better understanding of the general problem of irreversibility and of description of nonequilibrium systems. Typically, coexistence of an incoherent particlelike behavior, described by a quantum Boltzmann equation, and a wave-function description by means of Gross-Pitaevskii equation, is important for understanding Bose-Einstein condensation [24]. In Refs. [8,9] it was shown how the formalism we used in the present paper copes with the more general problem of nonequilibrium macroscopic systems. However, a systematic treatment of irreversibility in the very similar problem of atomic interferometry involves QED and is a future challenge.
[1] H. Rauch, in Advances in Quantum Phenomena, Vol. 347 of NATO Advanced Science Institutes, Series B. Physics, edited by E. G. Beltrametti and J.-M. Lévy-Leblond (Plenum, New York, 1995), p. 113.

[2] H. Rauch, in Proceedings of the 3rd International Symposium on Foundations of Quantum Mechanics, edited by S. Kobayashi et al. (Physical Society of Japan, Tokyo, 1990), p. 3.

[3] V. F. Sears, Neutron Optics (Oxford University Press, Oxford, 1989).

[4] V. F. Sears, Can. J. Phys. 56, 1261 (1978).

[5] V. F. Sears, Phys. Rep. 82, 1 (1982).

[6] C. S. Adams, M. Siegel, and J. Mlynek, Phys. Rep. 240, 143 (1994)

[7] L. Lanz and B. Vacchini, Int. J. Theor. Phys. 36, 67 (1997).

[8] L. Lanz, O. Melsheimer, and B. Vacchini, in Quantum Com- munication, Computing, and Measurement, edited by $\mathrm{O}$. Hirota, A. S. Holevo, and C. M. Caves (Plenum, New York, 1997), pp. 339-353.

[9] L. Lanz and B. Vacchini, Int. J. Theor. Phys. (to be published).

[10] G. Lindblad, Commun. Math. Phys. 48, 119 (1976).

[11] L. van Hove, Phys. Rev. 95, 249 (1954).

[12] M. Lax, Rev. Mod. Phys. 23, 287 (1951).

[13] M. L. Goldberger and F. Seitz, Phys. Rev. 71, 294 (1974).

[14] H. Rauch, W. Treimer, and U. Bonse, Phys. Lett. A 57, 369 (1974).

[15] J. D. Jackson, Classical Electrodynamics (Wiley, New York, 1962).

[16] J. Vigué, Phys. Rev. A 52, 3973 (1995).

[17] J. Schmiedmayer et al., Phys. Rev. Lett. 74, 1043 (1995).

[18] V. F. Sears, Physica B 151, 156 (1988). 
[19] O. Halpern, Phys. Rev. 88, 1003 (1952).

[20] H. Rauch, J. Summhammer, M. Zawisky, and E. Jericha, Phys. Rev. A 42, 3726 (1990).

[21] J. Summhammer, H. Rauch, and D. Tuppinger, Phys. Rev. A 36, 4447 (1987).
[22] P. A. Egelstaff, An Introduction to the Liquid State (Clarendon, Oxford, 1992).

[23] L. Diósi, Europhys. Lett. . 30, 63 (1995).

[24] C. W. Gardiner and P. Zoller, Phys. Rev. A 55, 2902 (1997). 